\title{
Recovery of right ventricular function and strain in patients with ST-segment elevation myocardial infarction and concurrent chronic total occlusion
}

\author{
Anna van Veelen ${ }^{1}$. Joëlle Elias ${ }^{1}$. Ivo M. van Dongen ${ }^{1}$ - Loes P. C. Hoebers ${ }^{1} \cdot$ Bimmer E. P. M. Claessen ${ }^{1}$. \\ Truls Ramunddal $^{2} \cdot$ Peep Laanmets ${ }^{3} \cdot$ Erlend Eriksen $^{4} \cdot$ René J. van der Schaaf $^{5} \cdot$ Robin Nijveldt $^{6}$. \\ Jose P. S. Henriques ${ }^{1} \cdot$ Alexander Hirsch ${ }^{7,8}$ (D)
}

Received: 10 June 2021 / Accepted: 16 September 2021 / Published online: 23 September 2021

(c) The Author(s) 2021

\begin{abstract}
The right ventricle (RV) is frequently involved in ST-segment elevation myocardial infarction (STEMI) when the culprit or concurrent chronic total occlusion (CTO) is located in the right coronary artery (RCA). We investigated RV function recovery in STEMI-patients with concurrent CTO. In EXPLORE, STEMI-patients with concurrent CTO were randomized to CTO percutaneous coronary intervention (PCI) or no CTO-PCI. We analyzed 174 EXPLORE patients with serial cardiovascular magnetic resonance imaging RV data (baseline and 4-month follow-up), divided into three groups: CTO-RCA (CTO in RCA, culprit in non-RCA; $n=89$ ), IRA-RCA (infarct related artery [IRA] in RCA, CTO in non-RCA; $n=56$ ), and no-RCA (culprit and CTO not in RCA; $n=29$ ). Tricuspid annular plane systolic excursion (TAPSE), RV ejection fraction (RVEF), RV global longitudinal strain (GLS) and free wall longitudinal strain (FWLS) were measured. We found that RV strain and TAPSE improved in IRA-RCA and CTO-RCA (irrespective of CTO-PCI) at follow-up, but not in no-RCA. Only RV FWLS was different among groups at baseline, which was lower in IRA-RCA than no-RCA $(-26.0 \pm 8.3 \%$ versus $-31.0 \pm 6.4 \%$, $\mathrm{p}=0.006$ ). Baseline RVEF, RV end-diastolic volume and TAPSE were associated with RVEF at 4 months. RV function parameters were not predictive of 4 year mortality, although RV GLS showed additional predictive value for New York Heart Association Classification $>1$ at 4 months. In conclusion, RV parameters significantly improved in patients with acute or chronic RCA occlusion, but not in no-RCA patients. RV FWLS was the only RV parameter able to discriminate between acute ischemic and non-ischemic myocardium. Moreover, RV GLS was independently predictive for functional status.
\end{abstract}

Keywords Right ventricle · ST-segment elevation myocardial infarction · Chronic total occlusion · Strain analysis · Cardiovascular magnetic resonance

Alexander Hirsch

a.hirsch@erasmusmc.nl

1 Department of Cardiology, Heart Center, Amsterdam UMC, University of Amsterdam, Amsterdam Cardiovascular Sciences, Amsterdam, The Netherlands

2 Department of Cardiology, Sahlgrenska University Hospital, Gothenburg, Sweden

3 Department of Cardiology, North-Estonia Medical Centre, Tallinn, Estonia

4 Department of Cardiology, Haukeland University Hospital, Bergen, Norway
5 Department of Cardiology, OLVG Hospital, Amsterdam, The Netherlands

6 Department of Cardiology, Radboud University Medical Center, Nijmegen, The Netherlands

7 Department of Cardiology, Erasmus Medical Center, University Medical Center Rotterdam, Room Rg-419, Dr. Molewaterplein 40, 3015 GD Rotterdam, The Netherlands

8 Department of Radiology and Nuclear Medicine, Erasmus Medical Center, University Medical Center Rotterdam, Rotterdam, The Netherlands 


\section{Introduction}

Assessment of the right ventricular (RV) function has prognostic implications in patients with ischemic heart disease [1]. The RV function can be expressed in global systolic function parameters, such as RV ejection fraction (RVEF) or tricuspid annular plane systolic excursion (TAPSE), but these parameters could overlook subtle and more regional dysfunction. A novel application of myocardial strain assessment using cardiovascular magnetic resonance imaging (CMR) derived myocardial feature-tracking (FT) enables advanced assessment of global and regional myocardial function [2]. Left ventricular strain analysis is able to accurately differentiate between infarcted and non-infarcted myocardium at segmental level [3]. Additionally, RV strain measurements are associated with global RV function and prognosis in patients with acute myocardial infarction [4-7].

In $15 \%$ of patients with an acute ST-segment elevation myocardial infarction (STEMI) a concomitant chronic total occlusion (CTO) is found-a $100 \%$ coronary lumen narrowing that is older than 3 months $[8,9]$. In these patients, the RV function is frequently affected when the right coronary artery (RCA) is the infarct-related artery (IRA) or the CTO-related artery [10]. The difference in RV function and RV strain recovery between these patients with either acute or chronic ischemia of the RV, has never been described. Separately, it is assumed that RV dysfunction after STEMI may recover within a few months [11]. Moreover, RV dysfunction resulting from CTO RCA has also been thought to be able to improve [12]. Whether this is due to successful revascularization of CTO RCA, the development of a collateral network or to a temporal effect of hibernation has not yet been established.

The Evaluating Xience and left ventricular function in PCI on occlusiOns afteR STEMI (EXPLORE) trial randomized STEMI patients with a concurrent non-IRA CTO to additional CTO PCI or no CTO PCI after successful primary PCI [13]. We used the serial CMR data of EXPLORE trial participants who underwent CMR at baseline and at 4 months to evaluate the recovery of RV function parameters (global function as well as regional strain) from baseline to 4 month follow-up in patients with either an acute or chronic RCA occlusion, and to determine the prognostic value of the RV function parameters on prognosis. Furthermore, we aimed to determine the treatment effect of CTO PCI RCA on RV function recovery when compared to a control group of no CTO PCI.

\section{Methods}

\section{Study design}

The study design and outcomes of the EXPLORE trial have been published previously [13]. In short, 302 STEMI patients with concurrent CTO in non-IRA were randomly assigned to CTO PCI within 7 days after primary PCI ('CTO PCI group') or no-CTO PCI for at least 4 months ('no-CTO PCI group'). To assess the primary endpoint of LV function, all patients underwent CMR at 4 months and preferably also at baseline. Clinical follow-up was obtained at 4 months and thereafter yearly until 5 years, and included functional status (angina and dyspnea) and survival status. Patients with factors hampering appropriate CMR, such as the presence of atrial fibrillation or cardiac devices at baseline, were excluded, as well as patients with hemodynamic instability $>48 \mathrm{~h}$ after STEMI. All inclusion and exclusion criteria have been published previously [14]. No significant difference was found for the primary endpoints of LVEF or LV end-diastolic volume between both treatment arms.

In the current substudy, we analyzed all patients who underwent baseline and follow-up CMR and excluded all patients without assessable RV images. The flowchart of patient selection is displayed in Fig. 1. A total of 180 patients underwent serial CMR imaging $[15,16]$. In 6 of these patients, no RV analysis could be done at baseline or follow-up, the remaining 174 patients were included in this study. For the purpose of this study, we divided these 174 patients into three groups: (1) CTO-RCA (patients with CTO in RCA and culprit in non-RCA), (2) IRA-RCA (culprit in RCA and CTO in non-RCA), and (3) no-RCA (culprit and CTO in non-RCA). The CTO-RCA group consisted of patients that were randomly assigned to CTO PCI and patients that were assigned to no CTO PCI. All angiographies were evaluated by an independent angiography core lab. A CTO was defined as a $100 \%$ luminal narrowing without antegrade flow [8]. The collateral quality was scored using the Rentrop score [17]. Rentrop score 0 and 1 were considered as poorly-developed collaterals while Rentrop score 2 and 3 were considered as well-developed. The study endpoints were (1) the recovery of RV function parameters, i.e. RVEF, TAPSE, RV global longitudinal strain and RV free-wall longitudinal strain, from baseline to 4 months follow-up, (2) the impact of revascularization of the CTO on RV global function and RV strain recovery, and (3) the prognostic value of RV function and strain. 


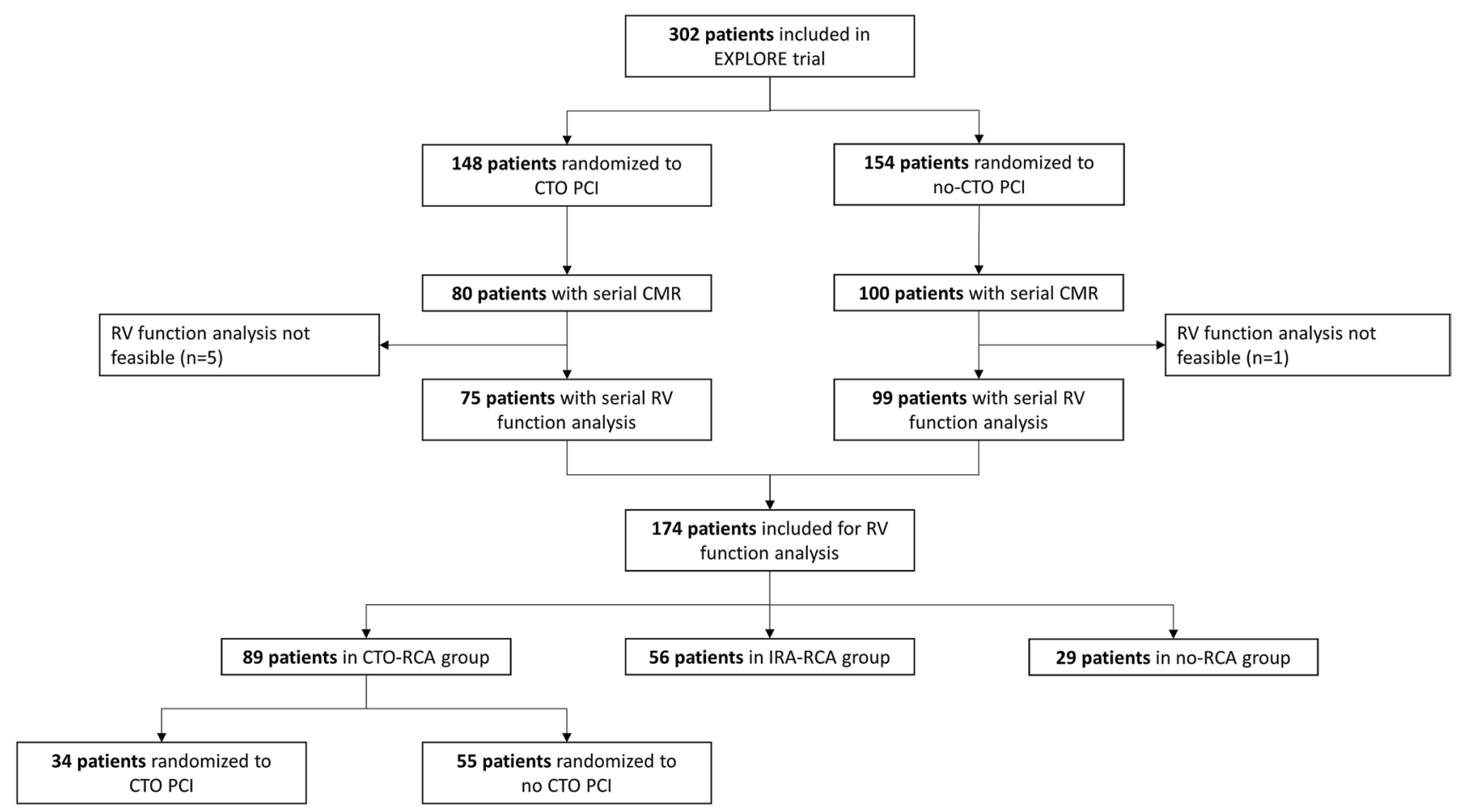

Fig. 1 Flowchart of patient selection. CMR cardiovascular magnetic resonance; $C T O$ chronic total occlusion; IRA infarct-related artery; $P C I$ percutaneous coronary intervention; $R C A$ right coronary artery; $R V$ right ventricle

\section{Left ventricular CMR analysis}

The imaging protocol and strain measurement technique have been published previously [15]. In short, electrocardiogram-gated steady-state free-precession cine images were obtained during repeated breath holds on 1.5-Tesla scanners. Late gadolinium enhanced images were used to identify the extent of the infarction. Left ventricular volumes have been measured previously for the primary outcome of the original EXPLORE study by an independent core laboratory, blinded for randomization outcome (ClinFact Corelab using Qmass MR analytical software version 7.6, Medis Medical Imaging Systems, Leiden, The Netherlands). Left ventricular myocardial longitudinal and circumferential strain measurement technique were described previously [15].

\section{Right ventricular CMR analysis}

Right ventricular myocardial strain was measured blinded for randomization outcome in the 4 chamber longitudinal axis images using commercially available FT-CMR software (QStrain, Medis Medical Imaging Systems version 2.0.12.2, Leiden, The Netherlands) under the supervision of an experienced CMR cardiologist (JE, supervision AH). The right ventricular endocardial contours were drawn manually during the end-diastolic and end-systolic phase. Subsequently, the software automatically traced the cardiac contours during the cardiac cycle, resulting in the peak global longitudinal strain (GLS) of the entire right ventricle, GLS of the RV free wall (FWLS) and GLS of the septum. Since deformation of the intraventricular septum is considered to be mainly affected by LV dysfunction, we chose to focus on the total RV GLS and the RV FWLS, rather than GLS of the septum [18]. RV volumes were measured in the short axis images and corrected for body-surface area $(\mathrm{AvV}$, supervision AH). For the calculation of the TAPSE, we measured the RV length in the 4 chamber longitudinal axis from the apex to the juncture of the tricuspid annulus with the RV free wall in end-diastole (end-diastolic length; EDL) and end-systole (end-systolic length; ESL), as described previously [19]. Then, the TAPSE was calculated by subtracting the ESL from the EDL. An example of the measurement techniques is displayed in Fig. 2.

\section{Reproducibility analysis}

We tested the intra- and interreproducibility of RV GLS, FWLS, TAPSE and RVEF measurements in 30 randomly selected scans. The measurements were repeated by the initial observer to measure the intraobserver variability and by a second observer to measure the interobserver variability, all blinded for the previous analyses. 


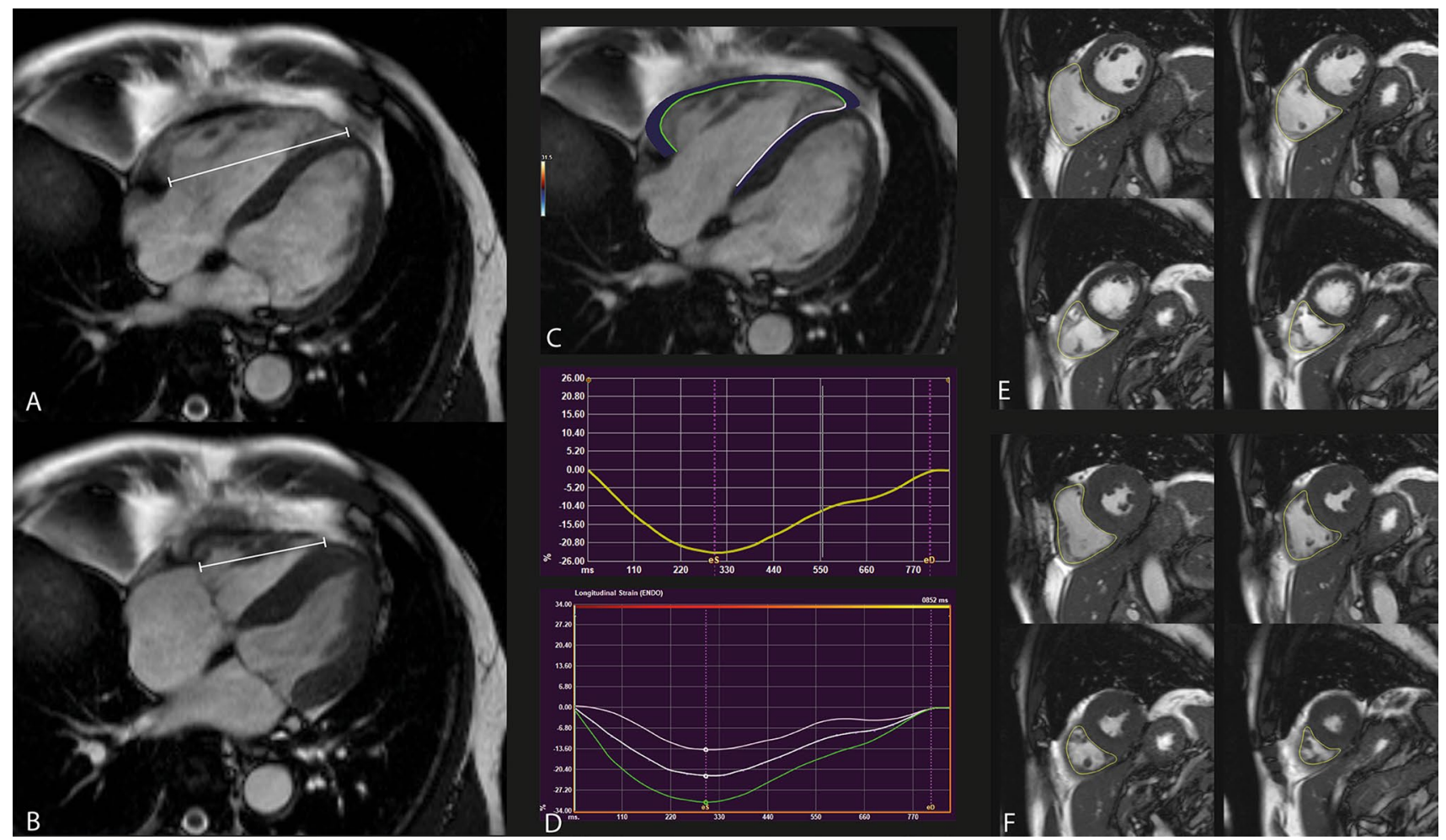

Fig. 2 Measurement of right ventricular function parameters. A and B End-diastolic length (A) and end-systolic length $(\mathbf{B})$ measurement for the calculation of the tricuspid annular plane systolic excursion; C Right ventricular strain measurement using feature tracking, with the green line indicating tracking of the free wall and white line indicating the septum tracking; D Strain curves, with the upper curve

\section{Statistical analysis}

Statistical analyses were performed using SPSS Statistics version 25. Categorical data were described as frequencies with percentages and compared using the Fisher's exact test to compare 2 groups or the Chi square test to compare $>2$ groups. The normality of numerical data was assessed by evaluation of the histogram and in case of doubt using the Kolmogorov-Smirnoff test. Normally distributed numerical data were described as mean \pm standard deviation $(\mathrm{SD})$ and compared using the Student's t-test for independent comparisons between 2 groups, the paired t-test for the comparison of 2 serial measurements within a group, and the one-way ANOVA for the independent comparison between 3 groups. When an overall significant ANOVA was found, post-hoc analyses were performed to identify subgroup differences. Pearson's correlation coefficient analysis and scatter plots were used to study the correlation between the different RV function parameters. Multivariable linear regression was used to identify predictors for RVEF at 4 month followup and multivariable logistic regression was used to identify predictors for New York Heart Association (NYHA) indicating the general RV strain curve and the lower curve indicating the RV strain curves divided into septum (white), average (white) and free wall (green). eS indicates end-systolic and $\mathrm{eD}$ indicates end-diastolic; E, F Right ventricular contours in the short-axis end-diastole (E) and end-systole (F) for RV volume measurements

Classification $>1$ at 4 month follow-up. Using Cox proportional hazards models we tested the effect of RV GLS and RV FWLS on mortality and calculated hazard ratios (HR) with 95\% confidence intervals (CI). Stepwise forward selection of variables was used for the regression models. Variables entered the models if $\mathrm{p}<0.05$ and were removed if $\mathrm{p}>0.10$. Inter- and intraobserver variability were calculated as intra-class correlations (ICC) using a model of absolute agreement. All tests were 2 -sided and a p-value of $<0.05$ was considered statistically significant.

\section{Results}

\section{Baseline characteristics}

The baseline characteristics of the included 174 patients are displayed in Table 1 . The mean age was $60 \pm 10$ years and $87 \%$ was male $(\mathrm{n}=151)$. Baseline CMR was performed at a median of 3 days (IQR 2-5) after primary PCI. Patients were divided into the three groups according to RCA involvement: (1) CTO-RCA $(\mathrm{n}=89) ; 2)$ IRA-RCA $(\mathrm{n}=56) ; 3)$ no-RCA 
Table 1 Baseline characteristics

\begin{tabular}{|c|c|c|c|c|c|}
\hline & $\begin{array}{l}\text { Total } \\
\mathrm{N}=174\end{array}$ & $\begin{array}{l}\text { no-RCA } \\
\mathrm{N}=29\end{array}$ & $\begin{array}{l}\text { CTO-RCA } \\
\mathrm{N}=89\end{array}$ & $\begin{array}{l}\text { IRA-RCA } \\
\mathrm{N}=56\end{array}$ & p-value ${ }^{a}$ \\
\hline \multicolumn{6}{|l|}{ Baseline characteristics } \\
\hline Age, years & $60 \pm 10$ & $61 \pm 10$ & $59 \pm 10$ & $60 \pm 10$ & 0.84 \\
\hline Male gender & $151(87)$ & $27(93)$ & $79(89)$ & $45(80)$ & 0.19 \\
\hline Diabetes mellitus & $27(16)$ & $6(21)$ & $14(16)$ & $7(13)$ & 0.61 \\
\hline Hypertension & $76(44)$ & $12(41)$ & $42(47)$ & $22(39)$ & 0.62 \\
\hline Hypercholesterolemia & $59(34)$ & $13(45)$ & $27(30)$ & $19(34)$ & 0.36 \\
\hline Current smoker & $90(52)$ & $13(45)$ & $47(53)$ & $30(54)$ & 0.72 \\
\hline Previous myocardial infarction & $25(14)$ & $7(24)$ & $15(17)$ & $3(5)$ & 0.041 \\
\hline Previous percutaneous coronary intervention & $18(10)$ & $5(17)$ & $10(11)$ & $3(5)$ & 0.22 \\
\hline Familial history of coronary artery disease & $70(40)$ & $11(38)$ & $37(42)$ & $22(39)$ & 0.93 \\
\hline \multicolumn{6}{|l|}{ Angiographic characteristics } \\
\hline Multiple CTOs & $20(12)$ & $2(7)$ & $14(16)$ & $4(7)$ & 0.20 \\
\hline Japan-CTO score & $2.1 \pm 1.1$ & $2.0 \pm 0.9$ & $2.4 \pm 1.1$ & $1.8 \pm 1.1$ & 0.010 \\
\hline Poorly-developed collaterals to the CTO & $75(43)$ & $14(48)$ & $28(32)$ & $33(59)$ & 0.004 \\
\hline Proximal location of CTO & $132(76)$ & $10(35)$ & $85(96)$ & $37(66)$ & $<0.001$ \\
\hline IRA location & & & & & $<0.001$ \\
\hline Left main/left anterior descending artery & $75(43)$ & $20(69)$ & $55(62)$ & - & \\
\hline Left circumflex artery & $43(25)$ & $9(31)$ & $34(38)$ & - & \\
\hline Right coronary artery & $56(32)$ & - & - & $56(100)$ & \\
\hline Proximal location of IRA & $135(78)$ & $20(69)$ & $61(69)$ & $54(96)$ & $<0.001$ \\
\hline Three-vessel disease & $71(41)$ & $9(31)$ & $37(42)$ & $25(45)$ & 0.47 \\
\hline SYNTAX Score after Primary PCI & $27 \pm 9$ & $30 \pm 9$ & $26 \pm 9$ & $27 \pm 9$ & 0.09 \\
\hline \multicolumn{6}{|l|}{ CMR characteristics } \\
\hline \multicolumn{6}{|l|}{ Right ventricle } \\
\hline End-diastolic volume, $\mathrm{ml} / \mathrm{m}^{2}$ & $77 \pm 20$ & $73 \pm 16$ & $77 \pm 20$ & $78 \pm 20$ & 0.59 \\
\hline End-systolic volume, $\mathrm{ml} / \mathrm{m}^{2}$ & $37 \pm 12$ & $35 \pm 12$ & $36 \pm 12$ & $38 \pm 13$ & 0.48 \\
\hline Ejection fraction, $\%$ & $52 \pm 9$ & $53 \pm 7$ & $53 \pm 9$ & $51 \pm 9$ & 0.44 \\
\hline TAPSE, mm & $19.2 \pm 5.4$ & $20.0 \pm 5.7$ & $19.6 \pm 5.4$ & $18.3 \pm 5.2$ & 0.26 \\
\hline Global longitudinal strain total, $\%$ & $-21.2 \pm 6.0$ & $-22.9 \pm 5.9$ & $-21.3 \pm 5.9$ & $-20.2 \pm 6.0$ & 0.15 \\
\hline Global longitudinal strain free wall, $\%$ & $-28.0 \pm 7.9$ & $-31.0 \pm 6.4$ & $-28.3 \pm 7.7$ & $-26.0 \pm 8.3$ & 0.018 \\
\hline Global longitudinal strain septum, $\%$ & $-14.4 \pm 6.6$ & $-16.1 \pm 6.6$ & $-13.7 \pm 6.6$ & $-14.6 \pm 6.5$ & 0.24 \\
\hline \multicolumn{6}{|l|}{ Left ventricle } \\
\hline End-diastolic volume, $\mathrm{ml} / \mathrm{m}^{2}$ & $102 \pm 23$ & $102 \pm 21$ & $104 \pm 22$ & $97 \pm 24$ & 0.07 \\
\hline End-systolic volume, $\mathrm{ml} / \mathrm{m}^{2}$ & $60 \pm 22$ & $62 \pm 19$ & $63 \pm 24$ & $55 \pm 22$ & 0.13 \\
\hline Ejection fraction, $\%$ & $42 \pm 11$ & $40 \pm 8$ & $41 \pm 13$ & $43 \pm 12$ & 0.59 \\
\hline Global longitudinal strain, $\%$ & $-14.2 \pm 6.4$ & $-10.7 \pm 5.0$ & $-13.8 \pm 6.3$ & $-16.5 \pm 6.2$ & 0.002 \\
\hline Global circumferential strain, $\%$ & $-19.7 \pm 7.0$ & $-18.7 \pm 5.4$ & $-18.9 \pm 7.2$ & $-21.2 \pm 7.2$ & 0.24 \\
\hline Infarct size (culprit, MVO, CTO), g & $11.8 \pm 10.7$ & $13.4 \pm 12.5$ & $13.3 \pm 12.1$ & $8.8 \pm 5.7$ & 0.07 \\
\hline Presence of MVO & $60(45)$ & $13(59)$ & $31(46)$ & $16(37)$ & 0.24 \\
\hline
\end{tabular}

Data are presented as numbers (percentages) or mean \pm standard deviation

$C M R$ cardiac magnetic resonance imaging; $C T O$ chronic total occlusion; IRA infarct-related artery; IQR interquartile range; $M V O$ microvascular obstruction; RCA right coronary artery; SYNTAX Score Synergy between PCI with Taxus and Cardiac Surgery Score; TAPSE tricuspid annular plane systolic excursion

${ }^{a}$ Outcomes were analyzed using the chi-square test for categorical data, the one-way ANOVA for normally distributed continuous data or the Kruskal-Wallis test for not-normally distributed continuous data

$(\mathrm{n}=29)$. There were no differences among the three groups regarding comorbidities. Patients in the CTO-RCA group had a higher Japan-CTO score (J-CTO) than patients in
IRA-RCA group $(2.4 \pm 1.1$ versus $1.8 \pm 1.1 ; \mathrm{p}=0.004)$, more frequently had well-developed collaterals (69\% versus $41 \%$ in the IRA-RCA group, $\mathrm{p}=0.002$ ) and a proximal location of 
the CTO (96\% versus $35 \%$ in the no-RCA group, $\mathrm{p}<0.001$, and $66 \%$ in the IRA-RCA group, $\mathrm{p}<0.001)$.

Baseline RV function was relatively preserved with a mean RVEF of $52 \% \pm 9$ and mean TAPSE of $19.2 \mathrm{~mm} \pm 5.4$. RVEF and TAPSE were not significantly different between the three groups. Baseline RV GLS was $-21.2 \pm 6.0 \%$ and RV FWLS $-28.0 \% \pm 7.9$. The only RV parameter that was different among RCA groups at baseline was RV FWLS, which was significantly lower in the IRA-RCA group $(-26.0 \% \pm 8.3)$ than in the no-RCA group $(-31.0 \% \pm 6.4$, $\mathrm{p}=0.006)$.

\section{RV function recovery}

Table 2 and Fig. 3 display the recovery of the different RV function parameters. RVEF improved significantly from baseline to 4 months follow-up in all groups. TAPSE improved only in the CTO-RCA $(\Delta 1.3 \mathrm{~mm} \pm 5.0, \mathrm{p}=0.018)$ and IRA-RCA groups $(\Delta 2.5 \mathrm{~mm} \pm 4.7, \mathrm{p}<0.001)$. The global RV GLS and RV FWLS improved significantly in the CTO-RCA group $(\Delta-2.1 \pm 6.7, \mathrm{p}=0.004$ and $\Delta-2.1 \pm 7.2$, $\mathrm{p}=0.008)$ and the IRA-RCA group $(\Delta-2.8 \pm 5.6, \mathrm{p}<0.001$ and $\Delta-3.0 \pm 8.4, \mathrm{p}=0.010)$, but not in the control group of no-RCA.

Within the total CTO-RCA group $(\mathrm{n}=89), 34$ patients were randomized to CTO PCI and 55 patients were randomized to no CTO PCI. Among these 34 patients the procedural success rate was $67.6 \%(n=23 / 34)$. The recovery of the RV function parameters RVEF, TAPSE and RV FWLS was comparable among randomization groups (Supplemental Table 1). In the CTO-RCA group, PCI of the CTO did not lead to improvement of the RV GLS or RV FWLS from baseline to follow-up.

\section{Correlation of RV function parameters}

Correlation between the three RV function parameters RVEF, TAPSE and RV FWLS was calculated. All combinations showed significant correlations. The scatterplots are displayed in Supplemental Fig. 1. The strongest correlation was found between TAPSE and RV FWLS (Pearson's $r=-0.56, \mathrm{p}<0.001)$.

\section{The prognostic value of RV function parameters}

In univariable linear regression, male gender, as well as all baseline RV function parameters, including global RV GLS and RV FWLS, were significant predictors for RVEF at 4-month follow-up. In stepwise forward selection of variables, baseline RVEF, baseline RVEDV and baseline TAPSE were independent predictors (Table 3).

In total, 8 of the 174 patients died (4.6\%) during a median follow-up of 4 years (IQR 2-5). The mortality rate was comparable between groups (1/29 no-RCA patients, 5/89

Table 2 Recovery of right ventricular function

\begin{tabular}{|c|c|c|c|c|c|c|}
\hline & & $\begin{array}{l}\text { Total } \\
\mathrm{N}=174\end{array}$ & $\begin{array}{l}\text { No-RCA } \\
\mathrm{N}=29\end{array}$ & $\begin{array}{l}\text { CTO-RCA } \\
\mathrm{N}=89\end{array}$ & $\begin{array}{l}\text { IRA-RCA } \\
\mathrm{N}=56\end{array}$ & p-value ${ }^{a}$ \\
\hline \multirow[t]{4}{*}{$\operatorname{RVEF}(\%)$} & Baseline & $52.4 \pm 8.5$ & $53.0 \pm 7.1$ & $53.0 \pm 8.9$ & $51.2 \pm 8.7$ & 0.44 \\
\hline & 4 months & $55.8 \pm 7.5$ & $55.9 \pm 5.4$ & $55.7 \pm 7.9$ & $55.8 \pm 7.7$ & $>0.99$ \\
\hline & Difference & $3.4 \pm 7.4$ & $2.8 \pm 6.2$ & $2.8 \pm 7.4$ & $4.6 \pm 8.0$ & 0.32 \\
\hline & p-value baseline vs 4 months ${ }^{\dagger}$ & $<0.001$ & 0.003 & $<0.001$ & $<0.001$ & \\
\hline \multirow[t]{4}{*}{ TAPSE (mm) } & Baseline & $19.2 \pm 5.4$ & $20.0 \pm 5.7$ & $19.6 \pm 5.4$ & $18.3 \pm 5.2$ & 0.26 \\
\hline & 4 months & $20.9 \pm 5.0$ & $21.6 \pm 4.5$ & $20.8 \pm 5.1$ & $20.8 \pm 5.3$ & 0.73 \\
\hline & Difference & $1.7 \pm 4.8$ & $1.6 \pm 4.5$ & $1.3 \pm 5.0$ & $2.5 \pm 4.7$ & 0.31 \\
\hline & $p$-value baseline vs 4 months ${ }^{\mathrm{b}}$ & $<0.001$ & 0.07 & 0.018 & $<0.001$ & \\
\hline \multirow[t]{4}{*}{ RV GLS (\%) } & Baseline & $-21.2 \pm 6.0$ & $-22.9 \pm 5.9$ & $-21.3 \pm 5.9$ & $-20.2 \pm 6.0$ & 0.15 \\
\hline & 4 months & $-23.4 \pm 5.2$ & $-23.8 \pm 4.3$ & $-23.4 \pm 5.7$ & $-23.1 \pm 4.8$ & 0.82 \\
\hline & Difference & $-2.1 \pm 6.3$ & $-0.9 \pm 6.2$ & $-2.1 \pm 6.7$ & $-2.8 \pm 5.6$ & 0.41 \\
\hline & p-value baseline vs 4 months ${ }^{\mathrm{b}}$ & $<0.001$ & 0.44 & 0.004 & $<0.001$ & \\
\hline \multirow[t]{4}{*}{ RV FWLS (\%) } & Baseline & $-28.0 \pm 7.9$ & $-31.0 \pm 6.4$ & $-28.3 \pm 7.7$ & $-26.0 \pm 8.3$ & 0.018 \\
\hline & 4 months & $-30.0 \pm 6.8$ & $-31.0 \pm 5.3$ & $-30.4 \pm 7.0$ & $-29.0 \pm 7.1$ & 0.35 \\
\hline & Difference & $-2.0 \pm 7.5$ & $0.0 \pm 6.6$ & $-2.1 \pm 7.2$ & $-3.0 \pm 8.4$ & 0.22 \\
\hline & -value baseline vs 4 months ${ }^{\mathrm{b}}$ & 0.001 & 0.99 & 0.008 & 0.010 & \\
\hline
\end{tabular}

$C T O$ chronic total occlusion; FWLS = free wall longitudinal strain; $G L S$ global longitudinal strain; IRA infarct-related artery; $R C A$ right coronary artery; $R V$ right ventricle; $R V E D V$ right ventricular end-diastolic volume; $R V E F$ right ventricular ejection fraction; TAPSE tricuspid annular plane systolic excursion

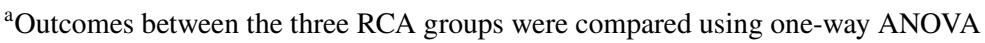

${ }^{\mathrm{b}}$ Outcomes between baseline and 4 months follow-up within groups was compared using the paired samples t-test 

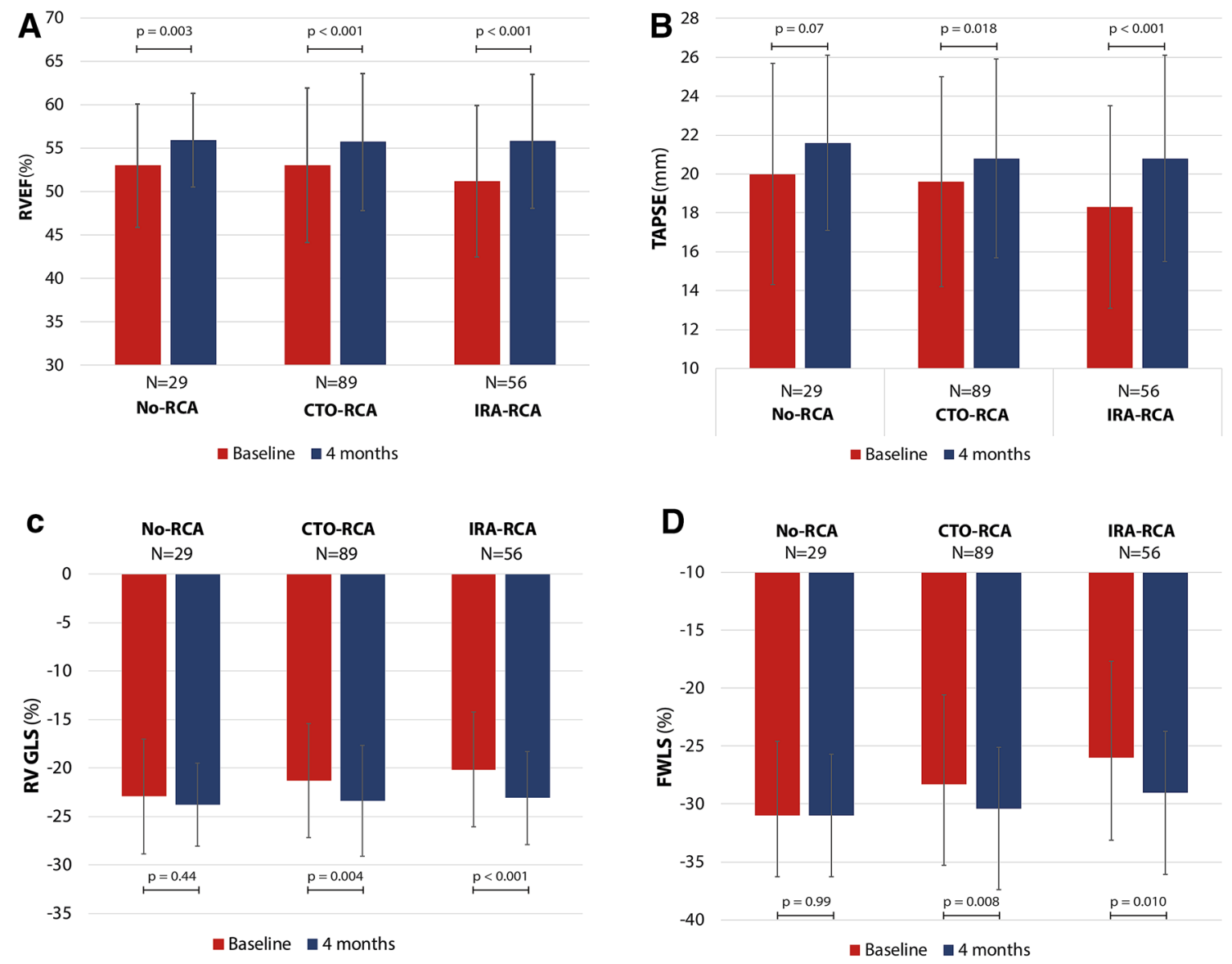

Fig. 3 Recovery of different right ventricular function parameters. A RVEF recovery; B TAPSE recovery; C RV global longitudinal strain recovery; D RV free wall longitudinal strain recovery. CTO chronic total occlusion; $F W L S$ free wall longitudinal strain; IRA infarct-

related artery; $P C I$ percutaneous coronary intervention; $R C A$ right coronary artery; $R V$ right ventricle; $R V E F$ right ventricular ejection fraction; TAPSE tricuspid annular plane systolic excursion

CTO-RCA patients and 2/56 IRA-RCA patients, $\mathrm{p}=0.81$ ). A multivariate cox proportional hazards model was created to test baseline and CMR characteristics as predictors for mortality (Supplemental Table 2). With a stepwise forward selection of variables, both age (HR 1.12 per year increment, 95\% CI 1.02-1.24, $\mathrm{p}=0.030$ ) and baseline LV GCS (HR $1.18,95 \%$ CI 1.02-1.35, $\mathrm{p}=0.022$ ) remained significant predictors. We tested the impact of RV CMR variables the clinical outcome NYHA classification. A total of $75.3 \%(\mathrm{n}=131)$ had NYHA I, $19.5 \%(\mathrm{n}=34)$ had NYHA II and $2.9 \%(n=5)$ had NYHA III at 4 month follow-up. In multivariable logistic regression, both RV GLS (OR 1.11, 95\% CI 1.01-1.21, $\mathrm{p}=0.024$ ) and LVEF (OR 0.94, 95\% CI $0.91-0.98, \mathrm{p}=0.002)$ were significantly associated with NYHA class II-III (Supplemental Table 3).

\section{Reproducibility}

The reproducibility of the RV parameters is demonstrated in Supplemental Table 4. The inter- and intraobserver

variability was 0.66 (95\% CI $0.01-0.88)$ and 0.73 (95\% CI $0.51-0.86)$ respectively for RVEF, and 0.80 (95\% CI $0.46-0.92)$ and 0.93 (95\% CI 0.86-0.97) for TAPSE. The RV strain measurements showed an excellent intraobserver variability (ICC for RV FWLS 0.89, 95\% CI 0.77-0.95; and ICC for RV GLS 0.83, 95\% CI 0.68-0.92) and interobserver variability for RV GLS (ICC $0.78,95 \%$ CI $0.58-0.89$ ), but a modest interobserver variability for RV FWLS (ICC 0.61, 95\% 0.19-0.82).

\section{Discussion}

In this CMR substudy of the EXPLORE trial, we evaluated the recovery and clinical value of RV function and RV strain parameters in patients with acute, chronic or no occlusion of the right coronary artery. We demonstrated that (1) RV free wall longitudinal strain significantly differed at baseline between the three groups with RV FWLS being the lowest in the patients with IRA-RCA compared to no-RCA, (2) 
Table 3 Multivariable linear regression model for the prediction of right ventricular ejection fraction at 4 months follow-up

\begin{tabular}{|c|c|c|c|c|c|c|c|c|c|}
\hline \multicolumn{5}{|c|}{ Univariable linear regression } & \multicolumn{5}{|c|}{ Multivariable linear regression } \\
\hline & \multirow[t]{2}{*}{ Beta } & \multicolumn{2}{|c|}{$95 \%$ confidence interval } & \multirow[t]{2}{*}{ p-value ${ }^{a}$} & & \multirow[t]{2}{*}{ Beta } & \multicolumn{2}{|c|}{$95 \%$ confidence interval } & \multirow[t]{2}{*}{ p-value* } \\
\hline & & Lower limit & Upper limit & & & & Lower limit & Upper limit & \\
\hline Age (years) & 0.07 & -0.04 & 0.18 & 0.23 & & & & & \\
\hline Male gender & -3.33 & -6.60 & -0.06 & 0.046 & & & & & \\
\hline Diabetes & 1.03 & -2.06 & 4.12 & 0.51 & & & & & \\
\hline CTO in RCA & 0.29 & -1.96 & 2.53 & 0.80 & & & & & \\
\hline IRA in RCA & 0.03 & -2.36 & 2.43 & 0.98 & & & & & \\
\hline $\begin{array}{l}\text { Randomisation to CTO } \\
\text { PCI }\end{array}$ & -1.20 & -3.45 & 1.06 & 0.30 & & & & & \\
\hline Baseline RVEF (\%) & 0.51 & 0.40 & 0.61 & $<0.001$ & Baseline RVEF (\%) & 0.43 & 0.32 & 0.55 & $<0.001$ \\
\hline $\begin{array}{l}\text { Baseline RVEDV ( } \mathrm{ml} / \\
\left.\mathrm{m}^{2}\right)\end{array}$ & -0.11 & -0.16 & -0.05 & $<0.001$ & $\begin{array}{l}\text { Baseline RVEDV (ml/ } \\
\mathrm{m}^{2} \text { ) }\end{array}$ & -0.11 & -0.16 & -0.06 & $<0.001$ \\
\hline Baseline RV GLS (\%) & -0.35 & -0.53 & -0.17 & $<0.001$ & & & & & \\
\hline Baseline RV FWLS (\%) & -0.31 & -0.44 & -0.17 & $<0.001$ & & & & & \\
\hline Baseline TAPSE (mm) & 0.35 & 0.15 & 0.55 & 0.001 & Baseline TAPSE (mm) & 0.20 & 0.01 & 0.39 & 0.044 \\
\hline
\end{tabular}

$C T O$ chronic total occlusion; IRA infarct-related artery; $R C A$ right coronary artery; $R V$ right ventricle; $R V E D V$ right ventricular end-diastolic volume; RVEF right ventricular ejection fraction; TAPSE tricuspid annular plane systolic excursion

${ }^{\mathrm{a}} \mathrm{A}$ stepwise forward selection of variables was used for multivariable linear regression

RV strain, RV ejection fraction and TAPSE significantly improved from baseline to 4 months follow-up in patients with an acute infarction in the RCA and in patients with a CTO in the RCA (irrespective of revascularization of the $\mathrm{CTO}$ ), and (3) RV global longitudinal strain was an independent predictor for functional dyspnea status at follow-up. However, RV strain was not predictive for RV ejection fraction or mortality in our cohort.

\section{RV strain}

Disruption of the coronary blood flow of more than $30 \mathrm{~min}$, in the setting of acute myocardial infarction, results in reduction of the myocardial contractility [20]. When the coronary hypoperfusion is transient due to restoration of the blood flow, this contractility may recover completely, although it can persist for several days-weeks after revascularization. This initial contractile dysfunction in the setting of restored blood flow is called "stunning", which is a defense mechanism of the cardiomyocytes to preserve the cardiac function in case of hypoxia, by downregulating the glucose uptake and oxidative metabolism [21]. Furthermore, contractile dysfunction could also exist in the setting of reduced coronary blood flow to the myocardium, which is called "hibernation", resulting from chronic hypoperfusion or after repetitive stunning [22]. Stunning and hibernation may occur locally in the myocardium subtended by the occluded artery, which in result, does not necessarily lead to impaired global function of the ventricle. This subclinical, regional dysfunction may be visualized with strain analysis. This technique allows the detection of minimal subtle myocardial deformation, that does not yet translate into global ventricular dysfunction. The tracing of myocardial borders to obtain strain measurements can be performed in echocardiography by speckle-tracking [23]. Feature-tracking strain measurement by CMR shows significant correlations to this previously established imaging technique, however, several studies imply that the modalities should not be used interchangeably but as substitute to the other [24,25]. The reproducibility of RV strain measurements showed good results for the intraobserver measurements (ICC 0.89 and 0.83 ), but was limited for inter-observer measurements (ICC 0.61 for RV FWLS). These numbers are comparable with previously described reproducibility rates for RV strain measured in Medis Medical Imaging software: intra-observer varying between 0.88 and 0.99 and inter-observer variability between 0.58 and 1.00 [25-27]. Additionally, RV strain measurements are susceptible to effects of training [26].

Indeed, we found that RV FWLS was significantly more impaired at baseline in the IRA-RCA group, compared to patients with no RCA involvement, while none of the global RV function parameters showed a significant difference between the three groups. This finding illustrates the additional value of RV strain measurement in the assessment of RV function, since it might detect early local deformation before the global ventricular function is affected. An alternative more rapid measurement technique for RV deformation is RV long-axis strain, which could be a complementary parameter for a complete RV functional assessment [28]. 


\section{$\mathrm{RV}$ function recovery}

The RV function (RVEF and TAPSE) and RV strain (RV GLS and RV FWLS) improved significantly from baseline to follow-up in patients in the IRA-RCA group and in patients in the CTO-RCA group (regardless of CTO revascularization). While the baseline LVEF was impaired in our study, the RV function parameters appeared to be within normal range at both baseline and follow-up. It should be noted that the preserved RV function parameters at baseline could have led to a reduced ability to find an impact of treatment on RV function recovery. Nonetheless, an improvement of the RV parameters was still observed. An improvement of RVEF was even observed in the no-RCA group, while none of the other RV parameters (TAPSE and RV strain) improved. We hypothesize that, although our study was limited by a low number of patients, the RV global function could be more congruent with the LV function and less affected by local deformation.

In patients with CTO-RCA, a previous observational echocardiography study found that the RV function (volumetric and strain) significantly improved 1 month after successful PCI of a CTO in the RCA, compared to baseline [12]. Contrarily, in our prospective randomized study, we found that RV function significantly improved in patients with a CTO-RCA, but this was irrespective of treatment allocation. It has been assumed that the vulnerability of the $\mathrm{RV}$ for persistent ischemic damage, in contrast to the $\mathrm{LV}$, is lower [29]. This is a result of a lower oxygen demand by the $\mathrm{RV}$ due to the thinner wall and lower afterload, and a higher potential for collateral circulation [30, 31]. Therefore, we postulate that a CTO in the RCA less frequently leads to persistent impairment of RV function, in contrast to the impact of a left-sided CTO on LV function. We demonstrated that the collateral network, associated with conservation of cardiac function [32], was better developed in patients in the CTO-RCA group compared to the other groups (with a CTO in the left anterior descending artery or circumflex artery). Hence, in these patients with a well-developed collateral circulation and preserved RV function, the additional effect of revascularization of a CTO on myocardial contractility is probably limited.

\section{Clinical implications}

In contrast to previous findings by Lu et al. [6], in our study baseline RV strain did not appear to be an independent predictor for RVEF at follow-up. Moreover, in speckle-tracking echocardiography studies by Park et al. [7] and Gavazzoni et al. [33], RV longitudinal strain was predictive for mortality and major adverse cardiovascular events or hospital admissions for heart failure. However, in our current CMR study, we found that neither RVEF nor RV strain were predictors for mortality, but our event numbers were low. It should be noted that the agreement of the different strain imaging modalities, using echocardiography as well as CMR, is limited [25], thus the modalities should probably not be used interchangeably.

As was suggested by several studies, RV strain may be used to predict functional outcome in patients with several etiologies of heart failure [7, 33-35]. To determine the prognostic value of RV function and strain on heart failure and functional outcome, we used NYHA class as a surrogate. A previous study described a correlation between RV strain and NYHA class [36]. In addition, we found that RV strain was independently associated with reduced functional status at 4 month follow-up. However, we could not confirm the previously described association with mortality $[4,7]$. This could partially be explained by the fact that this is a sub-analysis of the EXPLORE trial, which aimed to find differences after randomization to CTO PCI or no CTO PCI. Accordingly, the predefined study endpoints of the EXPLORE trial are mostly coronary events.

Strain analysis is an evolving technique that allows a quick and effortless evaluation of local deformation. RV strain showed significant correlation with the previously established RV functional parameters, RVEF and TAPSE, and could therefore also be of predictive value for clinical outcome, although this could not be demonstrated in our study due to low event numbers. Additionally, we found that strain was the only determinant that was able to discriminate between acute ischemic and non-ischemic myocardium, thus possibly has the ability to detect early local myocardial dysfunction. Strain analysis should probably not be considered a substitute for RVEF, but as an additional parameter for more complete RV function assessment.

\section{Limitations}

Some limitations should be acknowledged. First, we aimed to divide patients into groups that illustrated the RV involvement by IRA or CTO. However, by discriminating based on the presence of RCA disease, variations in coronary anatomy are not completely taken into account, since occlusions in the left anterior descending artery or the left circumflex artery may also lead to some RV involvement. Second, we tried to explore the predictive value of RV function on clinical follow-up, however, our sample size and event rates are too small to draw definite conclusions. Therefore, these results should be interpreted with caution and should be tested in a larger long-term cohort. The studied population had relatively preserved RV function thus strong associations on RV deformation would be hard to find. Third, the inter- and intraobserver reproducibility of the RV parameters is limited and potentially influenced by software type and no standards for FT-strain exist to date. 


\section{Conclusion}

In this RV CMR substudy of the EXPLORE trial, we demonstrated that RV strain, RVEF and TAPSE improved significantly from baseline to 4 months follow-up in patients with acute or chronic occlusion in the RCA. In an exploratory analysis, no treatment effect of PCI CTO of the RCA was found on RV function recovery. RV free wall longitudinal strain was, however, able to discriminate between acute ischemic and non-ischemic myocardium. RV strain did not have an additional predictive value on mortality, although limited by a low number of events. Still, RV strain was an additional predictor for functional status in terms of NYHA classification at 4 months follow-up.

Supplementary Information The online version contains supplementary material available at https://doi.org/10.1007/s10554-021-02423-9.

Author contributions All authors contributed to the study conception and design. AV, JE and AH: Material preparation, data collection and analysis were performed. AV, JE and AH: The first draft of the manuscript was written and all authors commented on previous versions of the manuscript. All authors read and approved the final manuscript.

Funding The EXPLORE study was investigator-initiated and funded by the Academic Medical Center and by a research grant from Abbott Vascular.

Data availability Upon request.

Code availability Not applicable.

\section{Declarations}

Conflict of interest All authors declare that they have no conflict of interest.

Consent to participate A verbal and written informed consent was obtained from all subjects.

Consent for publication All subjects signed consent for publication within the informed consent.

Ethical approval An ethics approval was obtained by the MEC AMC for the EXPLORE study.

Open Access This article is licensed under a Creative Commons Attribution 4.0 International License, which permits use, sharing, adaptation, distribution and reproduction in any medium or format, as long as you give appropriate credit to the original author(s) and the source, provide a link to the Creative Commons licence, and indicate if changes were made. The images or other third party material in this article are included in the article's Creative Commons licence, unless indicated otherwise in a credit line to the material. If material is not included in the article's Creative Commons licence and your intended use is not permitted by statutory regulation or exceeds the permitted use, you will need to obtain permission directly from the copyright holder. To view a copy of this licence, visit http://creativecommons.org/licenses/by/4.0/.

\section{References}

1. Zornoff LA, Skali H, Pfeffer MA et al (2002) Right ventricular dysfunction and risk of heart failure and mortality after myocardial infarction. J Am Coll Cardiol 39(9):1450-1455

2. Schuster A, Hor KN, Kowallick JT, Beerbaum P, Kutty S (2016) Cardiovascular magnetic resonance myocardial feature tracking: concepts and clinical applications. Circ Cardiovasc Imaging 9(4): 004077

3. Everaars H, Robbers LFHJ, Gotte M et al (2018) Strain analysis is superior to wall thickening in discriminating between infarcted myocardium with and without microvascular obstruction. Eur Radiol 28:5171-5181

4. Park JH, Negishi K, Kwon DH et al (2014) Validation of global longitudinal strain and strain rate as reliable markers of right ventricular dysfunction: comparison with cardiac magnetic resonance and outcome. J Cardiovasc Ultrasound 22(3):113-120

5. Truong VT, Safdar KS, Kalra DK et al (2017) Cardiac magnetic resonance tissue tracking in right ventricle: feasibility and normal values. Magn Reson Imaging 38:189-195

6. Lu KJ, Chen JX, Profitis K et al (2015) Right ventricular global longitudinal strain is an independent predictor of right ventricular function: a multimodality study of cardiac magnetic resonance imaging, real time three-dimensional echocardiography and speckle tracking echocardiography. Echocardiography 32(6):966-974

7. Park SJ, Park JH, Lee HS et al (2015) Impaired RV global longitudinal strain is associated with poor long-term clinical outcomes in patients with acute inferior STEMI. JACC Cardiovasc Imaging 8(2):161-169

8. Sianos G, Werner GS, Galassi AR et al (2012) Recanalisation of chronic total coronary occlusions: 2012 consensus document from the EuroCTO club. EuroIntervention 8(1):139-145

9. Claessen BE, van der Schaaf RJ, Verouden NJ et al (2009) Evaluation of the effect of a concurrent chronic total occlusion on long-term mortality and left ventricular function in patients after primary percutaneous coronary intervention. JACC Cardiovasc Interv 2(11):1128-1134

10. El Missiri AM, Guindy RR (2016) Echocardiographic assessment of right ventricular functions in patients with proximal right coronary artery chronic total occlusion. Int J Cardiovasc Imaging 32(6):895-903

11. Gorter TM, Lexis CP, Hummel YM et al (2016) Right ventricular function after acute myocardial infarction treated with primary percutaneous coronary intervention (from the glycometabolic intervention as adjunct to primary percutaneous coronary intervention in ST-segment elevation myocardial infarction III trial). Am J Cardiol 118(3):338-344

12. Ozkan B, Urumdas M, Alici G et al (2013) Echocardiographic evaluation of right ventricular functions after successful percutaneous recanalization of right coronary artery chronic total occlusions. Eur Rev Med Pharmacol Sci 17(7):917-922

13. Henriques JP, Hoebers LP, Ramunddal T et al (2016) Percutaneous intervention for concurrent chronic total occlusions in patients with STEMI: the EXPLORE trial. J Am Coll Cardiol 68(15):1622-1632

14. van der Schaaf RJ, Claessen BE, Hoebers LP et al (2010) Rationale and design of EXPLORE: a randomized, prospective, multicenter trial investigating the impact of recanalization of a chronic total occlusion on left ventricular function in patients after primary percutaneous coronary intervention for acute ST-elevation myocardial infarction. Trials 11:89

15. Elias J, van Dongen IM, Hoebers LP et al (2019) Recovery and prognostic value of myocardial strain in ST-segment elevation 
myocardial infarction patients with a concurrent chronic total occlusion. Eur Radiol 30:600-608

16. Elias J, van Dongen IM, Hoebers LP et al (2017) Improved recovery of regional left ventricular function after PCI of chronic total occlusion in STEMI patients: a cardiovascular magnetic resonance study of the randomized controlled EXPLORE trial. J Cardiovasc Magn Reson 19(1):53

17. Rentrop KP, Cohen M, Blanke H, Phillips RA (1985) Changes in collateral channel filling immediately after controlled coronary artery occlusion by an angioplasty balloon in human subjects. J Am Coll Cardiol 5(3):587-592

18. Molaug M, Stokland O, Ilebekk A, Lekven J, Kiil F (1981) Myocardial function of the interventricular septum. Effects of right and left ventricular pressure loading before and after pericardiotomy in dogs. Circ Res 49(1):52-61

19. Nijveldt R, Germans T, McCann GP, Beek AM, van Rossum AC (2008) Semi-quantitative assessment of right ventricular function in comparison to a 3D volumetric approach: a cardiovascular magnetic resonance study. Eur Radiol 18(11):2399-2405

20. Reimer KA, Lowe JE, Rasmussen MM, Jennings RB (1977) The wavefront phenomenon of ischemic cell death. 1. Myocardial infarct size vs duration of coronary occlusion in dogs. Circulation. 56(5):786-794

21. Di Carli MF, Prcevski P, Singh TP et al (2000) Myocardial blood flow, function, and metabolism in repetitive stunning. J Nucl Med 41(7):1227-1234

22. Schelbert HR (1998) Measurements of myocardial metabolism in patients with ischemic heart disease. Am J Cardiol 82(5a):61k-k67

23. Amzulescu MS, De Craene M, Langet H et al (2019) Myocardial strain imaging: review of general principles, validation, and sources of discrepancies. Eur Heart J Cardiovasc Imaging 20(6):605-619

24. Taha K, Bourfiss M, Te Riele A et al (2021) A head-to-head comparison of speckle tracking echocardiography and feature tracking cardiovascular magnetic resonance imaging in right ventricular deformation. Eur Heart J Cardiovasc Imaging 22(8):950-958

25. Erley J, Tanacli R, Genovese D et al (2020) Myocardial strain analysis of the right ventricle: comparison of different cardiovascular magnetic resonance and echocardiographic techniques. J Cardiovasc Magn Reson 22(1):51

26. Backhaus SJ, Metschies G, Billing M et al (2019) Cardiovascular magnetic resonance imaging feature tracking: impact of training on observer performance and reproducibility. PLoS ONE 14(1):e0210127
27. Gertz RJ, Lange T, Kowallick JT et al (2018) Inter-vendor reproducibility of left and right ventricular cardiovascular magnetic resonance myocardial feature-tracking. PLoS ONE 13(3):e0193746

28. Arenja N, Riffel JH, Djiokou CN et al (2016) Right ventricular long axis strain-validation of a novel parameter in non-ischemic dilated cardiomyopathy using standard cardiac magnetic resonance imaging. Eur J Radiol 85(7):1322-1328

29. Sanz J, Sanchez-Quintana D, Bossone E, Bogaard HJ, Naeije $R$ (2019) Anatomy, function, and dysfunction of the right ventricle: JACC state-of-the-art review. J Am Coll Cardiol 73(12):1463-1482

30. Kusachi S, Nishiyama O, Yasuhara K et al (1982) Right and left ventricular oxygen metabolism in open-chest dogs. Am J Physiol 243(5):H761-H766

31. Haupt HM, Hutchins GM, Moore GW (1983) Right ventricular infarction: role of the moderator band artery in determining infarct size. Circulation 67(6):1268-1272

32. van Dongen IM, Elias J, van Houwelingen KG et al (2018) Impact of collateralisation to a concomitant chronic total occlusion in patients with ST-elevation myocardial infarction: a subanalysis of the EXPLORE randomised controlled trial. Open Heart 5(2): $\mathrm{e} 000810$

33. Gavazzoni M, Badano LP, Vizzardi E et al (2020) Prognostic value of right ventricular free wall longitudinal strain in a large cohort of outpatients with left-side heart disease. Eur Heart J Cardiovasc Imaging 21(9):1013-1021

34. Hamada-Harimura Y, Seo Y, Ishizu T et al (2018) Incremental prognostic value of right ventricular strain in patients with acute decompensated heart failure. Circ Cardiovasc Imaging 11(10): e007249

35. Nunes Mdo C, Beloti FR, Lima MM et al (2010) Functional capacity and right ventricular function in patients with Chagas heart disease. Eur J Echocardiogr 11(7):590-595

36. Zhang S, Yang ZG, Sun JY et al (2014) Assessing right ventricular function in patients with hypertrophic cardiomyopathy with cardiac MRI: correlation with the New York Heart Function Assessment (NYHA) classification. PLoS ONE 9(9):e104312

Publisher's Note Springer Nature remains neutral with regard to jurisdictional claims in published maps and institutional affiliations. 